\title{
SCALE-UP OF MICROCHANNEL REACTORS FOR FISCHER-TROPSCH SYNTHESIS
}

\author{
Anna Lee Tonkovich*, Soumitra Deshmukh, Kai Jarosch, Luke Schrader, Sean \\ Fitzgerald, Dave Kilanowski, Jan Lerou, and Terry Mazanec \\ Velocys, Inc.
}

\begin{abstract}
Summary
The scale-up of a microchannel reactor for Fischer-Tropsch (FT) synthesis has progressed through multiple scales. Equivalent process performance was demonstrated across each scale. Small single channel test reactors ( 4 $\mathrm{cm}$ in length) have demonstrated excellent performance of an FT catalyst provided by Oxford Catalysts, Ltd. Catalyst from the same batch was tested in two long microchannel reactors ( $\sim 62 \mathrm{~cm}$ in length), with equal performance at a GHSV of $12,400 \mathrm{hr}^{-1}$. Further the same catalyst replicated the performance from both short and long single channel reactors in a pilot reactor with 276 parallel process channels ( $16 \mathrm{~cm}$ in length). The elusive premise of numbering up microchannel has been demonstrated, enabling the scale-up of reactor capacity.
\end{abstract}

Keywords

Scale-up, multichannel, Fischer-Tropsch synthesis, Numbering-up

\section{Introduction}

The early development of the FT process was undertaken by Franz Fischer and Hanz Tropsch in Germany in the 1920s and 1930s (1). The chemistry discovered by Fischer and Tropsch is based on creating long chain paraffinic hydrocarbons by polymerizing a mixture of carbon monoxide $(\mathrm{CO})$ and hydrogen $\left(\mathrm{H}_{2}\right)$, referred to as "synthesis gas", at an elevated pressure and temperature and in the presence of a cobalt catalyst. The reaction is highly exothermic and selectivity to the primary by-product, methane, is temperature sensitive thus control of catalyst bed temperature is important for high yield operation.

Over cobalt catalysts the primary products of FT synthesis are paraffins with a carbon number distribution, usually following the Anderson-Schulz-Flory distribution with the exception of carbon numbers less than $\mathrm{C} 4$. The reaction can be represented by the following chemical equation:

$$
\mathrm{nCO}+(2 \mathrm{n}+1) \mathrm{H}_{2} \rightarrow \mathrm{C}_{\mathrm{n}} \mathrm{H}_{2 \mathrm{n}+2}+\mathrm{H}_{2} \mathrm{O}
$$

Typical byproducts are liquefied petroleum gas (LPG) and naphtha. After the FT process, heavier hydrocarbons can be hydrocracked to produce distillate products, notably diesel and jet fuels (2). FT derived transportation fuels are typically referred to as synthetic fuels. Recently, there has been interest in synthetic fuels for four reasons: increasing world demand for fuels, the clean burning nature of synthetic fuels due to the absence of impurities (2), the increased desire to monetize stranded gas as energy prices rise, and the growing interest in producing renewable, environmentally-sustainable fuel from the gasification of non-edible biomass.

The demonstration of nearly isothermal performance of FT in a single microchannel has been described early in the literature by Wang and co-workers (2). A multichannel FT reactor was described by Jarosch and co-workers (3) and the performance was compared to predicted performance. This report compares the performance of multiple microchannel reactors evaluated with the identical F-T catalyst to clearly show how to scale-up the microchannel reactors. Neither channel length nor number of channels substantially changes the catalyst performance, when it is operated in a nearly isothermal microchannel reactor.

\footnotetext{
* To whom all correspondence should be addressed
} 


\section{Experimental and Results}

The scalability of microchannel reactors was demonstrated by testing four reactors using an identical catalyst (each reactor operated with fresh catalyst from the same batch). This study also addresses the effect of increasing channel length to increase productivity.

The first microchannel reactor (Short) had a characteristic dimension (gap) of $1 \mathrm{~mm}$, a width of $0.76 \mathrm{~cm}$ width and a catalyst bed length of $3.8 \mathrm{~cm}$ length. The second reactor (Long-A) contained one process microchannel with a dual gap of $1 \mathrm{~mm}$ for two-thirds of the channel width and $0.5 \mathrm{~mm}$ for one-third of the channel width. The channel width was $0.6 \mathrm{~cm}$ and the catalyst bed length was 61.6 $\mathrm{cm}$. The third reactor (Long B) had a catalyst bed length of $61.6 \mathrm{~cm}$, a width of $0.6 \mathrm{~cm}$ and a uniform gap of $1 \mathrm{~mm}$. The final reactor, (Pilot), had 276 process channels where each process channel had a dimension of $0.3 \mathrm{~cm}$ width, 1 $\mathrm{mm}$ gap, and $16.5 \mathrm{~cm}$ length. For the first three single channel reactors, heat was removed using hot oil flowing co-currently in microchannels flanking the process channel. The cooling medium employed in the pilot reactor was water maintained under partial boiling conditions. In the pilot reactor layers with catalyst containing process microchannels were flanked on either side by layers containing coolant microchannels. Process and coolant microchannels were oriented at with their major axes orthogonal to produce a cross-flow architecture.

Each reactor was loaded with a catalyst obtained from Oxford Catalysts, Ltd (OCL). The Co-based catalyst is based on the OCL patented OMX method for producing highly active and selective FT catalysts (5).

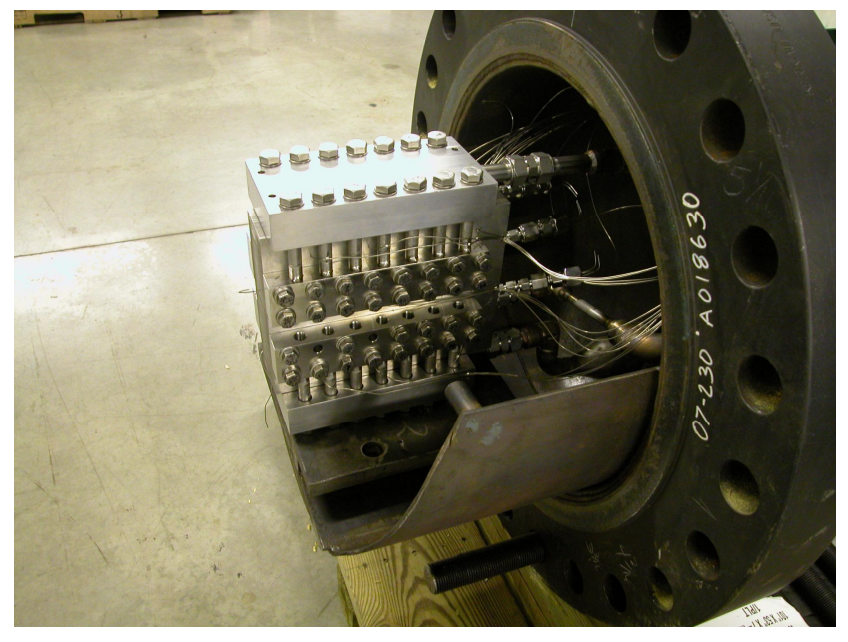

Figure 1. Pilot-scale FT Microchannel reactor with 276 parallel process channels and 132 coolant channels. The reactor is shown as it is installed in a pressure containment shell.
The operating conditions were varied for each reactor, but similar conditions were also tested across each reactor to validate scale-up in both length and number of channels. The FT feed was held constant with a 2:1 ratio of hydrogen to carbon monoxide and $16 \%$ diluent of nitrogen. The feed pressure inlet was held constant at 350 psig and the average reaction temperature was maintained at about $210 \mathrm{C}$. The feed gas flowrate was set to achieve a GHSV of 12,400 $\mathrm{hr}^{-1}$ for each of the four reactors. The results are shown in Figure 2.

For all four reactors the selectivity to methane averaged between $8 \%$ and $9 \%$ while the $\mathrm{CO}$ conversion ranged from $70 \%$ to $75 \%$ over the range of time-on-stream. The performance as a function of time-on-stream was similar across the four reactors and shows the natural deactivation of the FT process that is expected with the build up of waxes. Each reactor was regenerated and the performance was brought back to near initial levels.

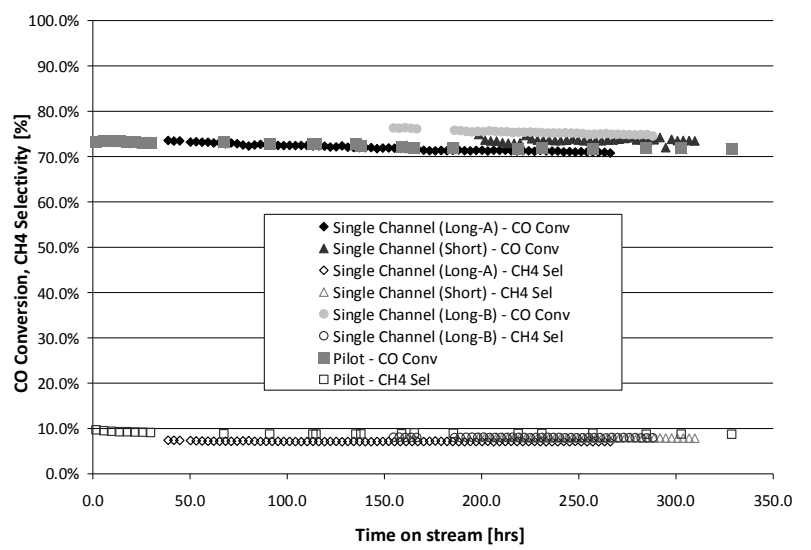

Figure 3. Performance comparison is shown for four microchannel reactors under equal reaction conditions.

\section{Conclusions}

The scalability of microchannel reactors was demonstrated experimentally using four reactors, demonstrating an increase in microchannel length and number of channels with equivalent performance.

\section{References}

(1) Strange, A. M.; AIChE 2003 Spring National Meeting, New Orleans, LA, 2003.

(2) Dry, M.E., High quality diesel via the Fischer-Tropsch process - a review, J Chem Technol Biotechnol, v77, pp4350, 2001

(3) Wang, Y, Vanderwiel, D., Tonkovich, A., Gao, Y., Baker, E., US 6,451,864, issued 2002

(4) Jarosch, K., Yang, B., Fitzgerald, S., Taha, R., Mazanec, T., Tonkovich, A., Reduced Complexity in the Representation of Fischer Tropsch Chemistry, Prepr. Pap.Am. Chem. Soc., Div. Fuel Chem, 2008, 53 (1), 1-2.

(5) Green, M., Xiao, T., US 20050250863 A1 